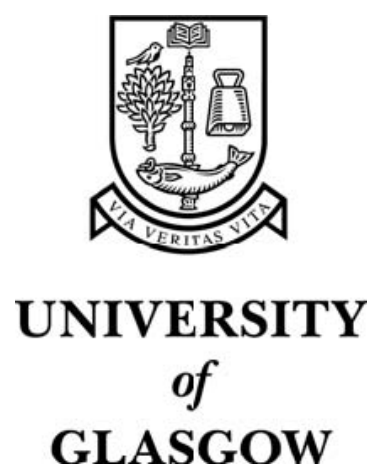

Vink, D. and Ballance, D. J. and Gawthrop, P. J. (2006) Bond graphs in model matching control. Mathematical and Computer Modelling of Dynamical Systems 12(2-3):pp. 103-105.

http://eprints.gla.ac.uk/2813/ 


\title{
Bond Graphs in Model Matching Control
}

\author{
DUSTIN VINK ${ }^{* 1}$ DONALD BALLANCE* AND PETER GAWTHROP*
}

\begin{abstract}
SUMMARY
Bond graphs are primarily used in the network modelling of lumped parameter physical systems, but controller design with this graphical technique is relatively unexplored. It is shown that bond graphs can be used as a tool for certain model matching control designs.

Some basic facts on the nonlinear model matching problem are recalled. The model matching problem is then associated with a particular disturbance decoupling problem, and it is demonstrated that bicausal assignment methods for bond graphs can be applied to solve the disturbance decoupling problem as to meet the model matching objective.

The adopted bond graph approach is presented through a detailed example, which shows that the obtained controller induces port-Hamiltonian error dynamics. As a result, the closed loop system has an associated standard bond graph representation, thereby rendering energy shaping and damping injection possible from within a graphical context.
\end{abstract}

Keywords: bond graph, bicausality, model matching, passivity, Hamiltonian.

\section{INTRODUCTION}

Henry Paynter introduced the bond graph methodology in 1959, and this graphical modelling technique has proven to be a convenient framework for the network modelling of lumped parameter physical systems. Bond graph theory has developed rapidly over the years and a variety of standard textbooks are now available; the reader is referred to the seminal book by Karnopp, Margolis and Rosenberg [9] for a comprehensive treatment on bond graph modelling.

It can be safely argued that the modelling capabilities of bond graphs are well understood. However, bond graph tools in control are considerably less explored, of which some results can be found in [4],[8], [14] and references therein. The shortage of literature on bond graph based control has compelled us to contribute bond graph tools for certain model matching problems. Of course, the authors are aware that passivitybased methods form a vast field in control design, so our account should be viewed as just one possible application of passivity concepts in physical model based control.

${ }^{1}$ Address correspondance to: Dustin Vink, d.vink@mech.gla.ac.uk

*Department of Mechanical Engineering, University of Glasgow, University Avenue, G12 8QQ, Glasgow, Scotland. 
This paper is organised as follows. Some essentials on the nonlinear Model Matching Problem (MMP) are addressed as detailed by Huijberts [7]. Then it is recalled that the MMP can be associated with a Disturbance Decoupling Problem (DDP), and to facilitate certain DDP solvability requirements, the DDP is formulated in terms of virtual actuation concepts.

Bicausal bond graphs, as introduced by Gawthrop [2], are used to find the controls that solve the associated DDP. It is shown that the controller induces port-Hamiltonian error dynamics such that a standard bond graph representation of this dynamics becomes possible. Further stabilisation is subsequently achieved through bond graph arguments. The reader is referred to [1], [5] and [13] for a comprehensive treatment on port-Hamiltonian systems.

To demonstrate the proposed methodology, a detailed example is presented. However, it was not attempted to derive a strict bond graph procedure for two main reasons. First, it is difficult to generalise in a nonlinear context, if not impossible. Second, the methodology considered here is based on the property that port-Hamiltonian error dynamics is induced by the controller, but it has not been verified whether this holds for general classes of bond graph models for which a particular MMP is solvable. Further research on this topic is required.

Now, it is certainly the case that bicausal bond graphs are not new and that they are widely used for many applications. On the other hand, the authors believe that the significance of this paper can be attributed to the explicit association of bicausal bond graphs with the DDP in the context of existing MMP theory. So, to the best of our knowledge, current applications of bicausal bond graphs in controller design have never been identified with the existing MMP theory as presented in [7] and references therein. The approach presented here could possibly spur, or perhaps renew, further research on bicausal bond graphs in physical model based control design.

\section{PRELIMINARIES}

Consider the nonlinear plant of the form

$$
P:\left\{\begin{array}{lll}
\dot{x} & = & f(x)+g(x) u \\
y & = & h(x)
\end{array}\right.
$$

where $x=\left(x_{1}, \ldots, x_{n}\right) \in \mathbb{R}^{n}$ are local coordinates, $u \in \mathbb{R}^{m}$ and $y \in \mathbb{R}^{p}$ are the system inputs and outputs respectively; $f(x)$ and $g(x)$ are smooth vector fields, and $h(x)$ is a smooth function. Furthermore, consider the nonlinear model of the form

$$
M:\left\{\begin{array}{lll}
\dot{\bar{x}} & = & \bar{f}(\bar{x})+\bar{g}(\bar{x}) \bar{u} \\
\bar{y} & = & \bar{h}(\bar{x})
\end{array}\right.
$$


where $\bar{x}=\left(\bar{x}_{1}, \ldots, \bar{x}_{\bar{n}}\right) \in \mathbb{R}^{\bar{n}}$ are local coordinates, $\bar{u} \in \mathbb{R}^{\bar{m}}$ and $\bar{y} \in \mathbb{R}^{p}$ are the model inputs and outputs respectively; $\bar{f}(\bar{x})$ and $\bar{g}(\bar{x})$ are smooth vector fields, and $\bar{h}(\bar{x})$ is a smooth function. Moreover, take $\bar{m} \leq m$ but observe that $P$ and $M$ have the same number of outputs.

Definition 2.1 (Nonlinear Model Matching Problem (MMP)). Consider the plant $P$ of the form (2.1) and the model $M$ of the form (2.2). The MMP is said to be locally solvable around $\left(x_{0}, \bar{x}_{0}\right) \in \mathbb{R}^{n} \times \mathbb{R}^{\bar{n}}$ if there exist controls $u(t)$ such that the plant outputs $y(t)$ track the model outputs $\bar{y}(t)$.

The above definition covers the essence of the MMP as described by Huijberts [7], in which two cases of the MMP are addressed: strong and weak model matching. The strong MMP has the control objective $|y(t)-\bar{y}(t)|=0$ for all $t \geq 0$ whereas the weak MMP aims at the convergence property $|y(t)-\bar{y}(t)| \rightarrow 0$ as $t \rightarrow \infty$. Note that $|\cdot|$ denotes the usual Euclidean norm. It can be said that strong model matching is achieved through proper controller initialisation, but this could be difficult to achieve in real applications. The weak MMP will therefore be considered in the following developments only.

Now, the MMP for $P$ and $M$ can be solved by solving its associated DDP [6]. To this end, one considers the extended system $E=P \times M$ defined as

$$
E:\left\{\begin{array}{l}
\dot{x}_{e}=f_{e}\left(x_{e}\right)+g_{e}\left(x_{e}\right) u+p_{e}\left(x_{e}\right) \bar{u} \\
y_{e}=h_{e}\left(x_{e}\right)
\end{array}\right.
$$

where $x_{e}=(x, \bar{x})$ and

$$
\begin{array}{ll}
f_{e}\left(x_{e}\right)=\left[\begin{array}{c}
f(x) \\
\bar{f}(\bar{x})
\end{array}\right], & g_{e}\left(x_{e}\right)=\left[\begin{array}{c}
g(x) \\
0
\end{array}\right] \\
p_{e}\left(x_{e}\right)=\left[\begin{array}{c}
0 \\
\bar{g}(\bar{x})
\end{array}\right], & h_{e}\left(x_{e}\right)=h(x)-\bar{h}(\bar{x}) .
\end{array}
$$

The key step in solving the MMP is to view the model inputs $\bar{u}$ as disturbances to the extended system $E$, so that solving the MMP turns into a DDP: The output $y_{e}\left(x_{e}\right)$ is to be rendered independent of $\bar{u}$ through the controls $u$, if possible. And to achieve output matching, the output $y_{e}\left(x_{e}\right)$ is to be stabilised to zero by further control. Huijberts [7] offers the following result on the solvability of the MMP.

Theorem 2.1. Consider the nonlinear plant $P$ and the nonlinear model $M$. Let the point $\left(x_{0}, \bar{x}_{0}\right) \in \mathbb{R}^{n} \times \mathbb{R}^{\bar{n}}$ be given. The MMP is locally solvable around $\left(x_{0}, \bar{x}_{0}\right)$ if and only if the non-regular dynamic disturbance decoupling with disturbance measurement is locally solvable around $\left(x_{0}, \bar{x}_{0}\right)$. 
The solvability requirements of various disturbance decoupling problems can be stated in terms of maximally controlled invariant distributions or algorithms [7]. These solvability requirements are not elaborated. However, for the DDP considered here, it generally suffices to require that relative degrees of model inputs $\bar{u}$ are not strictly less than the plant inputs $u$ with respect to the output $y_{e}\left(x_{e}\right)$. This requirement allows the disturbances $\bar{u}$ to be "intercepted" by the controls $u$ in many cases.

\section{SETTING UP THE NONLINEAR MODEL MATCHING PROBLEM}

This section defines a particular form of the MPP through the use of virtual actuator concepts in a physical model based context. It is not attempted to derive a strict bond graph approach, but a more heuristic approach is taken instead to retain flexibility in the design.

It is shown that bicausal bond graphs [2] can be used to solve the associated DDP by effectively inverting the plant such that controls for disturbance decoupling are readily found.

\subsection{Defining the plant bond graph}

To give physical structure to the general MMP as described earlier, the systems of both the plant (2.1) and model (2.2) are specified with bond graphs. To this end, a vector bond graph for $P$ depicted in Figure 1 is considered. The inputs and outputs are defined as

$$
\begin{array}{ll}
\tilde{u}^{1}=\left(\tilde{u}_{1}^{1}, \ldots, \tilde{u}_{m_{1}}^{1}\right), & \tilde{u}^{2}=\left(\tilde{u}_{1}^{2}, \ldots, \tilde{u}_{m_{2}}^{2}\right) \\
u^{1}=\left(u_{1}^{1}, \ldots, u_{p_{1}}^{1}\right), & u^{2}=\left(u_{1}^{2}, \ldots, u_{p_{2}}^{2}\right) \\
y^{1}=\left(y_{1}^{1}, \ldots, y_{p_{1}}^{1}\right), & y^{2}=\left(y_{1}^{2}, \ldots, y_{p_{2}}^{2}\right)
\end{array}
$$

such that $m_{1}+m_{2}+p_{1}+p_{2}=m$ and $p_{1}+p_{2}=p$. Note that the superscripts " 1 " and " 2 " associate their variables with flow and effort sources respectively. The total stored energy of the plant is denoted by the smooth Hamiltonian $H: \mathbb{R}^{n} \rightarrow \mathbb{R}$, which comprises the energy stored in $\mathbf{I}, \mathbf{C}$ and possibly multi-port elements.

The conjugate outputs of $\tilde{u}^{1}$ and $\tilde{u}^{2}$, being $\tilde{y}^{1}$ and $\tilde{y}^{2}$ respectively, will not play any role in the MMP considered here and have therefore been ignored. However, ignoring these outputs will lead to a plant $P$ that is not port-Hamiltonian: Standard bond graphs of which all conjugate input and output variables of source elements are taken into account belong to a class of port-Hamiltonian systems [5]. Nonetheless, it will be shown that ignoring the outputs $\tilde{y}^{1}$ and $\tilde{y}^{2}$ does not pose problems in the context of the paper, because the main objective is to have port-Hamiltonian error dynamics that allows for a standard bond graph representation. This will become clear in the sequel. 


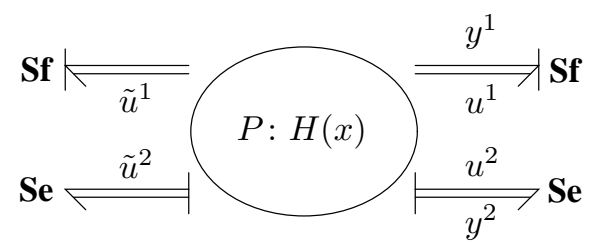

Fig. 1. Plant bond graph

\subsection{Defining the model bond graph}

Next consider the bond graph model of $M$ depicted in Figure 2, where the pairs $\left(\bar{u}^{1}, \bar{y}^{1}\right)$ and $\left(\bar{u}^{2}, \bar{y}^{2}\right)$ are defined as

$$
\begin{array}{ll}
\bar{u}^{1}=\left(\bar{u}_{1}^{1}, \ldots, \bar{u}_{p_{1}}^{1}\right), & \bar{u}^{2}=\left(\bar{u}_{1}^{2}, \ldots, \bar{u}_{p_{2}}^{2}\right) \\
\bar{y}^{1}=\left(\bar{y}_{1}^{1}, \ldots, \bar{y}_{p_{1}}^{1}\right), & \bar{y}^{2}=\left(\bar{y}_{1}^{2}, \ldots, \bar{y}_{p_{2}}^{2}\right) .
\end{array}
$$

Note that the number of model inputs and outputs is the same as the number of plant inputs and outputs $\left(u^{1}, y^{1}\right)$ and $\left(u^{2}, y^{2}\right)$. The total stored energy is denoted as the smooth Hamiltonian $\bar{H}: \mathbb{R}^{\bar{n}} \rightarrow \mathbb{R}$.

Let the bond graph of $M$ be identical to $P$ but without the $\tilde{u}^{1}$ and $\tilde{u}^{2}$ inputs. This is possible because of (3.1) and (3.2). The desired behavior of $y$ is then obtained by adding bond graph components to $M$, where the MMP will ensure that $y$ converges to $\bar{y}$, if solvable. The additive components of $M$ therefore act as virtual actuators that specify desired behavior for the plant outputs.

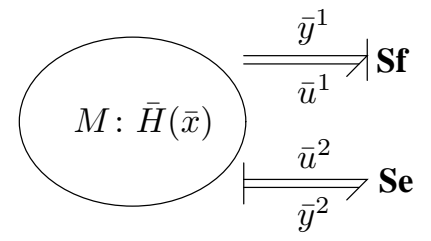

Fig. 2. Model bond graph

\subsection{Setting up the DDP}

Now that the bond graphs of $P$ and $M$ have been defined, the extended system (2.3) is readily obtained. To do this, first collect the input and output variables as

$$
\begin{aligned}
u & =\left(u^{1}, u^{2}, \tilde{u}^{1}, \tilde{u}^{2}\right), y & =\left(y^{1}, y^{2}\right) \\
\bar{u} & =\left(\bar{u}^{1}, \bar{u}^{2}\right), \quad \bar{y} & =\left(\bar{y}^{1}, \bar{y}^{2}\right) .
\end{aligned}
$$


Then by using the fact that standard bond graphs generate a class of port-Hamiltonian systems [5], it is assumed that the bond graphs of $P$ and $M$ generate the systems

$$
P:\left\{\begin{aligned}
\dot{x} & =[J(x)-R(x)] \mathbf{d} H(x)-g(x) u \\
y & =h(x) .
\end{aligned}\right.
$$

and

$$
M:\left\{\begin{aligned}
\dot{\bar{x}} & =[\bar{J}(\bar{x})-\bar{R}(\bar{x})] \mathbf{d} \bar{H}(\bar{x})-\bar{g}(\bar{x}) \bar{u} \\
\bar{y} & =\bar{h}(\bar{x}),
\end{aligned}\right.
$$

where $\mathbf{d} H(x)$ and $\mathbf{d} \bar{H}(\bar{x})$ are column gradient vectors; $J(x)=-J^{T}(x)$ and $R(x)=R^{T}(x)$ are $n \times n$ matrices, and $\bar{J}(\bar{x})=-\bar{J}^{T}(\bar{x})$ and $\bar{R}(\bar{x})=\bar{R}^{T}(\bar{x})$ are $\bar{n} \times \bar{n}$ matrices. In addition, both matrices $R(x)$ and $\bar{R}(\bar{x})$ are positive semidefinite.

In view of (2.3), the MPP can now be associated with a DDP by considering the extended system

$$
\begin{aligned}
{\left[\begin{array}{c}
\dot{x} \\
\dot{\bar{x}}
\end{array}\right] } & =\left[\begin{array}{cc}
J(x)-R(x) & 0 \\
0 & \bar{J}(\bar{x})-\bar{R}(\bar{x})
\end{array}\right]\left[\begin{array}{l}
\mathbf{d} H(x) \\
\mathbf{d} \bar{H}(\bar{x})
\end{array}\right]-\left[\begin{array}{c}
g(x) \\
0
\end{array}\right] u-\left[\begin{array}{c}
0 \\
\bar{g}(\bar{x})
\end{array}\right] \bar{u} \\
y_{e} & =h(x)-\bar{h}(\bar{x}) .
\end{aligned}
$$

From Theorem 2.1 it follows that the matching of $y$ with $\bar{y}$ is possible if and only if $y_{e}$ can be rendered independent of the model inputs $\bar{u}$ through the controls $u$.

\subsection{Solving the DDP and MMP}

In [7], various procedures for solving DDP's are addressed but these will not be recalled here. Nonetheless, it can be argued that many of these procedures have great similarities with feedback linearisation schemes for the extended system (2.3) of which the disturbances $\bar{u}$ do not have relative degrees strictly less than the relative degrees of the controls $u$.

The above consideration will proof to be quite effective in the context of the paper. First the plant is inverted as depicted in Figure 3. Then the relations $y=\bar{y}$ are enforced, and because inputs $\bar{u}$ are treated as measurable disturbances, the example will show that the relations $u^{1}=\bar{u}^{1}$ and $u^{2}=\bar{u}^{2}$ follow as to decouple $\bar{u}$. The controls $\tilde{u}^{1}$ and $\tilde{u}^{2}$ provide the remaining solutions to the DDP.

It is emphasised that solvability of the DDP considered here cannot be guaranteed by merely inverting the plant as in Figure 3 . However, since $M$ is structurally close to $P$ implies that causal paths from $\bar{y}$ to $\bar{u}$ are topologically similar to the causal paths from $y$ to $u$. It therefore seems intuitive plausible that relative degree conditions are more likely to be satisfied in this case, but further research on DDP solvability in relation to bicausal bond graph models is certainly needed. 
The MMP can be solved once the model inputs have been decoupled, so that further control on the decoupled extended system yields the desired decaying behavior of the extended output $y_{e}\left(x_{e}\right)$.

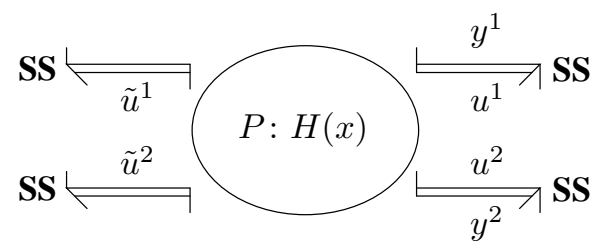

Fig. 3. Bicausal bond graph of plant $P$

\section{EXAMPLE}

This section demonstrates the application of bond graphs for the MMP as defined in Section 3. The example shows that bond graphs, in combination with virtual actuation concepts, can clarify certain physical model based control objectives.

\subsection{A mechanical system}

Consider the mechanical system depicted in Figure 4, of which the plant bond graph is depicted in Figure 5.

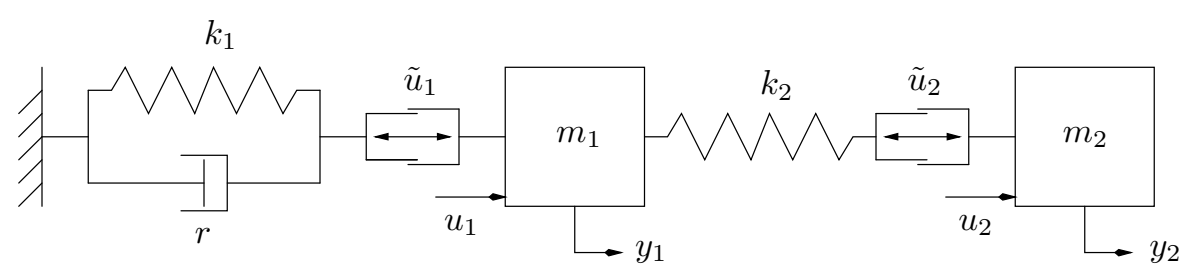

Fig. 4. Mechanical system

The plant inputs $u_{1}$ and $u_{2}$ denote forces whereas inputs $\tilde{u}_{1}$ and $\tilde{u}_{2}$ denote velocities; system outputs $y_{1}$ and $y_{2}$ are the respective velocities of masses $m_{1}$ and $m_{2}$; parameters $k_{1}$ and $k_{2}$ are spring stiffness constants, and $r$ is the damping coefficient.

Now, it could be desirable to specify desired port behavior for $\left(u_{1}, y_{1}\right)$ and $\left(u_{2}, y_{2}\right)$ in terms of a known physical system through control on $\tilde{u}_{1}$ and $\tilde{u}_{2}$. As a result, the control problem tends to be more physically motivated, thereby making the case for physical model based controllers and their possible significance. Such an approach turns out to be possible for the above system in the MMP context of Section 3. 


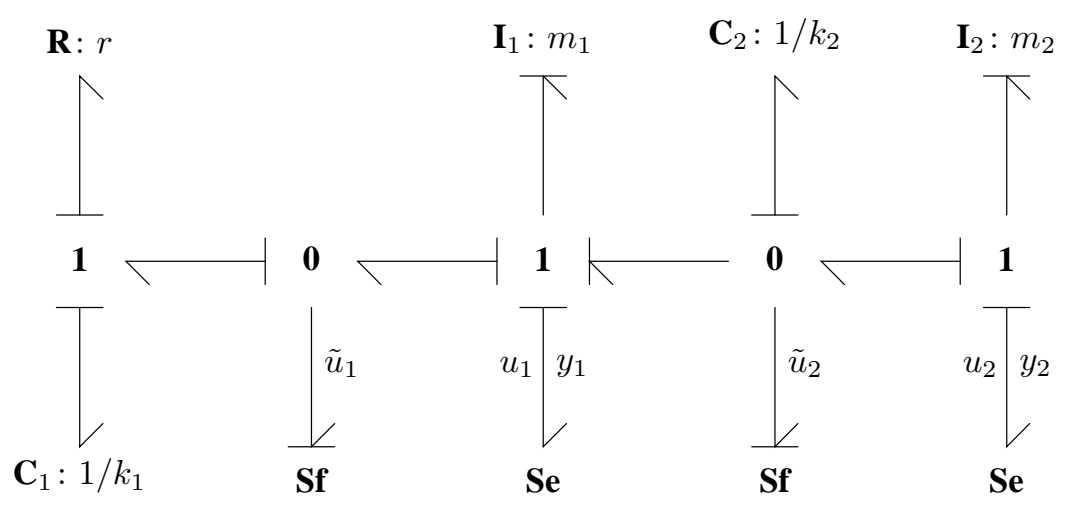

Fig. 5. Mechanical system bond graph

To this end, let $\left(x_{1}, \ldots, x_{4}\right)$ be local coordinates on $\mathcal{X}=\mathbb{R}^{4}$ that correspond to the storage components $\mathbf{C}_{1}, \mathbf{C}_{2}, \mathbf{I}_{1}$ and $\mathbf{I}_{2}$ respectively. From the causal assignment it readily follows that the plant $P$ takes the form

$$
\begin{aligned}
{\left[\begin{array}{c}
\dot{x}_{1} \\
\dot{x}_{2} \\
\dot{x}_{3} \\
\dot{x}_{4}
\end{array}\right] } & =\left[\begin{array}{cccc}
0 & 0 & 1 & 0 \\
0 & 0 & -1 & 1 \\
-1 & 1 & -r & 0 \\
0 & -1 & 0 & 0
\end{array}\right] \mathbf{d} H(x)-\left[\begin{array}{cccc}
0 & 0 & 1 & 0 \\
0 & 0 & 0 & 1 \\
1 & 0 & -r & 0 \\
0 & 1 & 0 & 0
\end{array}\right] u \\
y & =\left[\begin{array}{llll}
0 & 0 & 1 & 0 \\
0 & 0 & 0 & 1
\end{array}\right] \mathbf{d} H(x)
\end{aligned}
$$

where $u^{T}=\left(u_{1}, u_{2}, \tilde{u}_{1}, \tilde{u}_{2}\right)$ and where the Hamiltonian $H: \mathcal{X} \rightarrow \mathbb{R}$ is defined as

$$
H(x)=\frac{1}{2}\left(k_{1} x_{1}^{2}+k_{2} x_{2}^{2}+\frac{1}{m_{1}} x_{3}^{2}+\frac{1}{m_{2}} x_{4}^{2}\right) .
$$

\subsection{The model}

Suppose that the port pairs $\left(u_{1}, y_{1}\right)$ and $\left(u_{2}, y_{2}\right)$ of $P$ are to match the port pairs of some model $M$ as defined in Section 3.2. To do this, consider the model in Figure 6 and its associated bond graph in Figure 7.

The main idea behind the MMP as applied to this example becomes evident by removing $\tilde{u}_{1}$ and $\tilde{u}_{2}$ from $P$ such that $M$ becomes equivalent to $P$ once $\mathbf{C}_{3}$ of $M$ has been removed. Hence, the $\mathbf{C}_{3}$ component is seen to virtually actuate $P$ through the controls $\tilde{u}_{1}$ and $\tilde{u}_{2}$, which can be considered to be working in the background as to attain the desired port behavior for $\left(u_{1}, y_{1}\right)$ and $\left(u_{2}, y_{2}\right)$. 


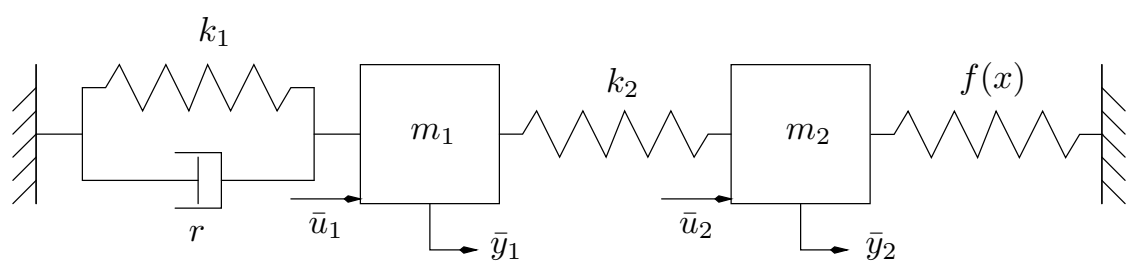

Fig. 6. Mechanical model

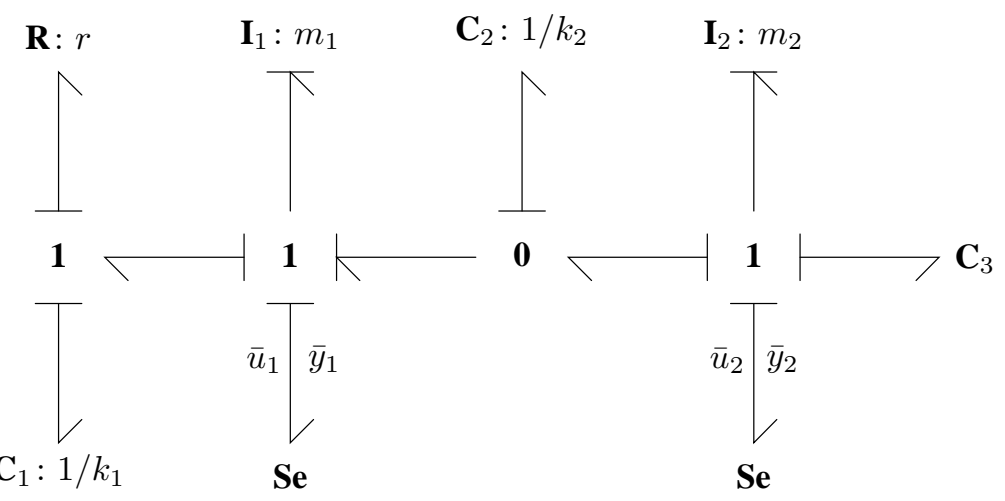

Fig. 7. Mechanical model bond graph

Let $\left(\bar{x}_{1}, \ldots, \bar{x}_{5}\right)$ be local coordinates on $\overline{\mathcal{X}}=\mathbb{R}^{5}$ that correspond to the $\mathbf{C}_{1}, \mathbf{C}_{2}, \mathbf{I}_{1}, \mathbf{I}_{2}$ and $\mathbf{C}_{3}$ elements respectively, then standard causal analysis yields the system

$$
\begin{aligned}
{\left[\begin{array}{c}
\dot{\bar{x}}_{1} \\
\overline{\bar{x}}_{2} \\
\dot{\bar{x}}_{3} \\
\overline{\bar{x}}_{4} \\
\dot{\bar{x}}_{5}
\end{array}\right] } & =\left[\begin{array}{ccccc}
0 & 0 & 1 & 0 & 0 \\
0 & 0 & -1 & 1 & 0 \\
-1 & 1 & -r & 0 & 0 \\
0 & -1 & 0 & 0 & -1 \\
0 & 0 & 0 & 1 & 0
\end{array}\right] \mathbf{d} \bar{H}(\bar{x})-\left[\begin{array}{ll}
0 & 0 \\
0 & 0 \\
1 & 0 \\
0 & 1 \\
0 & 0
\end{array}\right] \bar{u} \\
\bar{y} & =\left[\begin{array}{lllll}
0 & 0 & 1 & 0 & 0 \\
0 & 0 & 0 & 1 & 0
\end{array}\right] \mathbf{d} \bar{H}(\bar{x})
\end{aligned}
$$

where $\bar{u}^{T}=\left(\bar{u}_{1}, \bar{u}_{2}\right)$ and where the Hamiltonian $\bar{H}: \overline{\mathcal{X}} \rightarrow \mathbb{R}$ is defined as

$$
\bar{H}(\bar{x})=\frac{1}{2}\left(k_{1} \bar{x}_{1}^{2}+k_{2} \bar{x}_{2}^{2}+\frac{1}{m_{1}} \bar{x}_{3}^{2}+\frac{1}{m_{2}} \bar{x}_{4}^{2}\right)+\cosh \left(\bar{x}_{5}\right) .
$$

Observe that the nonlinear spring force of $\mathbf{C}_{3}$ is given by $f\left(\bar{x}_{5}\right)=\sinh \left(\bar{x}_{5}\right)$. 


\subsection{Solving the DPP}

The DDP is now solved in view of the bicausal bond graph arguments of Section 3.4. To this end, there is no need to construct the extended system (3.6) explicitly since the feedback linearisation scheme is applied to $P$ only.

Now, the inverse of $P$ can be conveniently depicted and obtained from Figure 8 , which is an important step in the approach considered here.

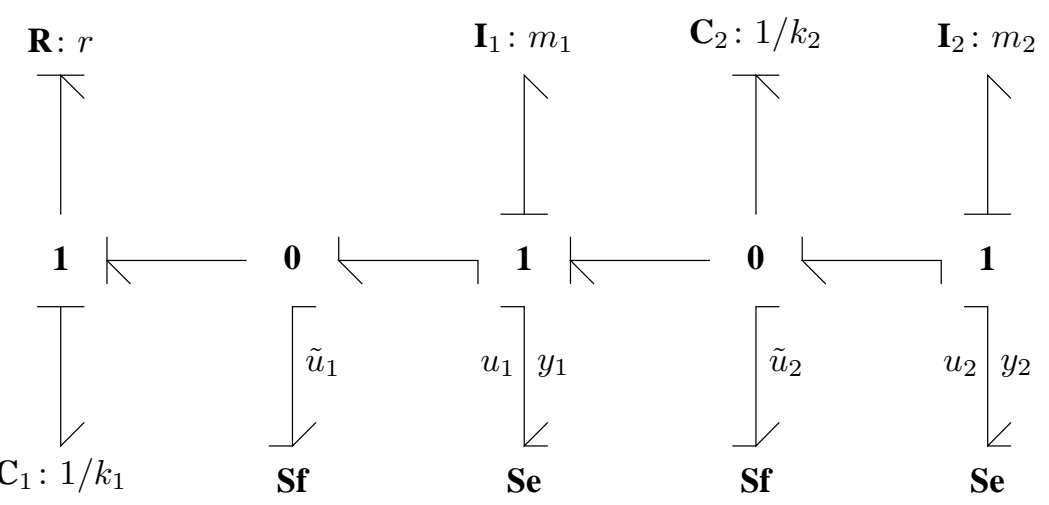

Fig. 8. Bicausal plant bond graph

The bond graph inversion of $P$ is particularly instructive since the bicausal bond graph shows the existence of one-dimensional internal dynamics: The $\mathbf{C}_{1}$ component retains its integral causality after bicausal assignment [3]. From Figure 8 it readily follows that the inverse of $P$ takes the form

$$
\begin{aligned}
\dot{\eta} & =-\tilde{u}_{1}+y_{1} \\
\tilde{u}_{1} & =y_{1}+\frac{1}{r}\left(m_{1} \dot{y}_{1}+m_{2} \dot{y}_{2}+k_{1} \eta+u_{1}+u_{2}\right) \\
\tilde{u}_{2} & =y_{2}-y_{1}+\frac{m_{2}}{k_{2}} \ddot{y}_{2}+\frac{1}{k_{2}} \dot{u}_{2},
\end{aligned}
$$

where $\eta$ denotes the internal dynamics. In case the reader is not familiar with bicausal bond graphs, applying the constrained dynamics algorithm described in [11] to the constraints $y_{1}-x_{3} / m_{1}=0$ and $y_{2}-x_{4} / m_{2}=0$ will yield the inverse system (4.5). 
The decoupling controller is found by enforcing the relation $y=\bar{y}$ such that the time derivatives of $y$ in (4.5) are taken along trajectories of $M$. Doing so yields the decoupling controller

$$
\begin{aligned}
\dot{\bar{x}} & =[\bar{J}(\bar{x})-\bar{R}(\bar{x})] \mathbf{d} \bar{H}-\bar{g}(\bar{x}) \bar{u} \\
\dot{\eta} & =-\tilde{u}_{1}+\frac{1}{m_{1}} \bar{x}_{3} \\
u_{1} & =\bar{u}_{1} \\
u_{2} & =\bar{u}_{2} \\
\tilde{u}_{1} & =-\frac{k_{1}}{r} \bar{x}_{1}-\frac{1}{r} \sinh \left(\bar{x}_{5}\right)+\frac{k_{1}}{r} \eta-\frac{1}{r} \mu_{1} \\
\tilde{u}_{2} & =-\frac{1}{k_{2} m_{2}} \bar{x}_{4} \cosh \left(\bar{x}_{5}\right)+\mu_{2}
\end{aligned}
$$

where $\mu_{1}$ and $\mu_{2}$ are newly introduced controls. Note that a copy of $M$ has been added since the controller states depend on $\bar{x}$, so that the order of dynamic compensation is correlated to the dimension of the reference model.

Claim 4.1. The controller (4.6) induces port-Hamiltonian error dynamics, which therefore admits a standard bond graph representation.

Proof. By uniqueness and existence of trajectories, each state of the system (4.1) has an associated "error" variable $e_{i}(t)=x_{i}(t)-x_{i}^{r}(t)$ where $x_{i}^{r}(t)$ are reference signals. Consider the relations

$$
\begin{aligned}
e_{1} & =x_{1}-\eta, & e_{3} & =x_{3}-\bar{x}_{3} \\
e_{2} & =x_{2}-\bar{x}_{2}-\frac{1}{k_{2}} \sinh \left(\bar{x}_{5}\right), & e_{4} & =x_{4}-\bar{x}_{4} .
\end{aligned}
$$

By using (4.1), (4.3) and (4.6) it can be verified that the error dynamics with coordinates $\left(e_{1}, \ldots, e_{4}\right)$ on $\mathcal{E}=\mathbb{R}^{4}$ takes the form

$$
\begin{aligned}
{\left[\begin{array}{c}
\dot{e}_{1} \\
\dot{e}_{2} \\
\dot{e}_{3} \\
\dot{e}_{4}
\end{array}\right] } & =\left[\begin{array}{cccc}
0 & 0 & 1 & 0 \\
0 & 0 & -1 & 1 \\
-1 & 1 & -r & 0 \\
0 & -1 & 0 & 0
\end{array}\right] \mathbf{d} H_{e}(e)-\left[\begin{array}{ll}
0 & 0 \\
0 & 1 \\
1 & 0 \\
0 & 0
\end{array}\right] \mu \\
\psi & =\left[\begin{array}{llll}
0 & 0 & 1 & 0 \\
0 & 1 & 0 & 0
\end{array}\right] \mathbf{d} H_{e}(e),
\end{aligned}
$$

where $\mu^{T}=\left(\mu_{1}, \mu_{2}\right)$ and $\psi^{T}=\left(\psi_{1}, \psi_{2}\right)$ are the new inputs and outputs respectively. The Hamiltonian $H_{e}: \mathcal{E} \rightarrow \mathbb{R}$ is given by

$$
H_{e}(e)=\frac{1}{2}\left(k_{1} e_{1}^{2}+k_{2} e_{2}^{2}+\frac{1}{m_{1}} e_{3}^{2}+\frac{1}{m_{2}} e_{4}^{2}\right)
$$


Observe that (4.8) is port-Hamiltonian, so that there exists a standard bond graph representation [5]. In addition, it is seen that the structure of (4.8) is close to (4.1), so it is readily seen that (4.8) admits the bond graph in Figure 9.

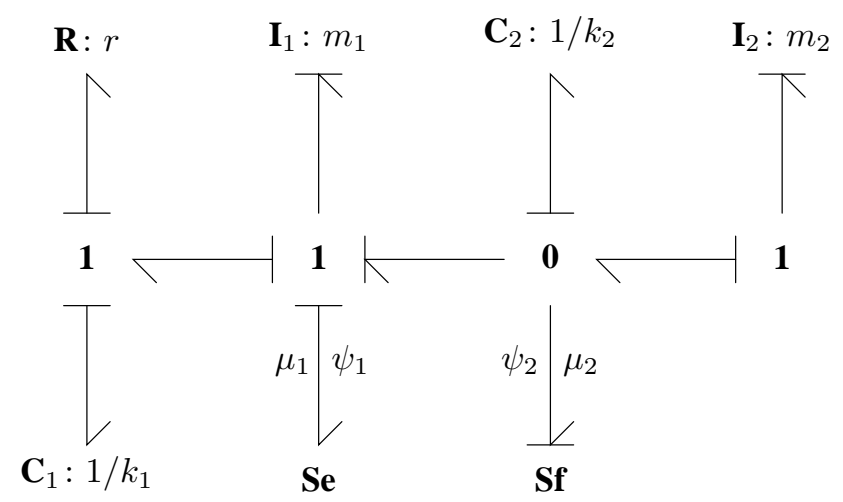

Fig. 9. Error dynamics

\subsection{Solving the MMP}

Now that the controller (4.6) has been found that solves the DDP, it remains to be shown how the extended output $y_{e}\left(x_{e}\right)$ can be stabilised to zero.

Claim 4.2. The equilibrium point $e=0$ of (4.8) is globally asymptotically stable for $\mu=0$, and, as a result, the controller (4.6) solves the MMP.

Proof. Let $\mu=0$ and write (4.8) as

$$
\begin{gathered}
\dot{e}=f(e)-g v \\
{\left[\begin{array}{c}
\dot{e}_{1} \\
\dot{e}_{2} \\
\dot{e}_{3} \\
\dot{e}_{4}
\end{array}\right]=\left[\begin{array}{cccc}
0 & 0 & 1 & 0 \\
0 & 0 & -1 & 1 \\
-1 & 1 & 0 & 0 \\
0 & -1 & 0 & 0
\end{array}\right] \mathbf{d} H_{e}(e)-\left[\begin{array}{l}
0 \\
0 \\
1 \\
0
\end{array}\right] v,}
\end{gathered}
$$

where $v=r L_{g} H_{e}(e)$ and where $L_{g}$ denotes the standard Lie derivative. The reason for writing (4.8) in the form (4.11) is that this allows Theorem 10.9 in [11] to be used for addressing the asymptotic behavior of the origin.

Next consider the relation

$$
\frac{d}{d t} H_{e}(e)=-\frac{r}{m_{1}^{2}} e_{3}^{2}=-r\left[L_{g} H_{e}(e)\right]^{2},
$$


which follows from Figure 9 by equating the sum of all outgoing power flows to zero. Global stability is therefore implied since $H_{e}$ is positive definite and radially unbounded.

The global asymptotic stability of the origin can now be established through a standard LaSalle argument [10]. To this end, let $Z$ be a compact set containing the origin and let $e(t) \in Z$ for all $t \geq 0$. In addition, define the set

$$
B=\left\{e \in \mathbb{R}^{4}: L_{g} H_{e}(e)=0\right\} .
$$

From (4.12) it follows that the system (4.11) converges to the largest invariant set contained in $Z \cap B$. Then, by taking (4.11) and (4.13) into account, Theorem 10.9 in [11] concludes that $\{0\}$ is the largest invariant set contained in $Z \cap B$. The origin is therefore globally asymptotically stable and the weak MMP objective $|y(t)-\bar{y}(t)| \rightarrow 0$ for $t \rightarrow \infty$ is attained.

Even though the controller (4.6) with $\mu=0$ yields a globally decaying extended output $y_{e}\left(x_{e}\right)$, further control on $\mu$ is necessary to improve the response time. To this end, consider the $\mathbf{S e}$ and $\mathbf{S f}$ elements in Figure 9 and replace them by linear $\mathbf{R}$ components, leading to the controls $\mu_{1}=d_{1} \psi_{1}$ and $\mu_{2}=d_{2} \psi_{2}$ for some positive damping constants $d_{1}$ and $d_{2}$. When this control is applied, the new dissipation rate becomes

$$
\frac{d}{d t} H_{e}(e)=-d_{2} k_{2}^{2} e_{2}^{2}-\frac{r+d_{1}}{m_{1}^{2}} e_{3}^{2} .
$$

In view of Passivity-Based Control (PBC), it is known that damping injection alone need not improve performance and that the energy often needs to be shaped to improve response times [12]. Loosely speaking, PBC comes down to finding some desired energy function such that its time derivative can be rendered non-positive through the controls. But despite the conceptual clarity of PBC, it can be a difficult undertaking for certain systems, especially in a nonlinear context. Nonetheless, no difficulties exist for the simple example considered here since the bond graph in Figure 9 readily reveals certain attainable energy shaping.

To see how the energy can be shaped by using Figure 9, observe that $\mu_{1}$ can be associated with the output of $\mathbf{C}_{1}$ whereas $\mu_{2}$ can be associated with the output of $\mathbf{I}_{2}$. Next consider the error dynamics (4.8) and its shaped Hamiltonian $H_{s}(e)$ defined as

$$
H_{s}(e)=H_{e}(e)+\frac{1}{2}\left(c_{1} e_{1}^{2}+c_{2} e_{4}^{2}\right),
$$

where $c_{1}$ and $c_{2}$ are positive constants. Taking the time derivative along the trajectories of (4.8) yields

$$
\frac{d}{d t} H_{s}(e)=-\frac{1}{m_{1}} e_{3}\left(\mu_{1}-c_{1} e_{1}\right)-k_{2} e_{2}\left(\mu_{2}+c_{2} e_{4}\right)-\frac{r}{m_{1}^{2}} e_{3}^{2} .
$$

Hence, the controls $\mu_{1}=c_{1} e_{1}$ and $\mu_{2}=-c_{2} e_{4}$ render (4.16) non-positive. 


\section{CONCLUDING REMARKS}

Bicausal bond graphs were used to solve a physical model based disturbance decoupling problem. The inverted plant, in bicausal sense, was used to find those controls that decoupled the model inputs. This methodology was based on the simple requirement that the relative degrees of the model inputs should not be strictly less than the relative degrees of the plant inputs. It was seen that this relative degree condition was likely to be fulfilled in case the reference model was geometrically "close" to the plant. However, it was not attempted to derive general solvability requirements for the DDP considered in the paper, so that more research on bicausal bond graphs in relation to the DDP is required.

An important aspect of bicausal bond graphs, as applied in the paper, is that the inverse dynamics is required to remain stable for arbitrary model outputs that are to be tracked. This is not a mere shortcoming of bicausal bond graphs but an intrinsic problem when inverse systems are used for tracking control designs. Also, it is known that controllers based on inverse dynamics often lack certain robustness requirements. It is our intention to address internal stability and robustness issues of bond graph based controllers in future work.

Finally, it is important to note that the proposed bond graph methodology cannot be guaranteed to work at all times, mainly because the MMP setup was rather heuristic in nature, thereby retaining flexibility in case of unexpected difficulties. Nonetheless, the authors believe that the paper presented a useful bond graph approach for physical model based control using passivity and model matching techniques.

\section{REFERENCES}

1. Dalsmo, M., van der Schaft, A. J. On representations and integrability of mathematical structures in energy-conserving physical systems. SIAM Journal on Control and Optimization, 37(1):54-91, 1998.

2. Gawthrop, P. J. Bicausal bond graphs. In Cellier and Granda, pages 83-88, 1995.

3. Gawthrop, P. J. Physical interpretation of inverse dynamics using bicausal bond graphs. Journal of the Franklin Institute, 337:743-769, 2000.

4. Gawthrop, P. J., Ballance, D., Vink, D. Bond graph based control with virtual actuators. In Simulation in Industry. ESS, 2001.

5. Golo G., A.J. van der Schaft, P.C. Breedveld and B.M. Maschke. Hamiltonian formulation of bond graphs, pages 1-2. Lecture Notes for the Euro/Geoplex Summer School. Springer-Verlag, 2003.

6. Huijberts, H. J. C. A nonregular solution of the nonlinear dynamic disturbance decoupling problem with an application to a complete solution of the nonlinear model matching problem. SIAM Journal on Control and Optimization, 30:336-349, 1992.

7. Huijberts, H. J. C. Dynamic Feedback in Nonlinear Synthesis Problems. Centrum voor Wiskunde en Informatica, 1994.

8. Junco, S. Lyapunov second method and feedback stabilization directly on bond graphs. In Proceedings of the ICBGM, 2001. 
9. Karnopp, D. C., Margolis, D., Rosenberg, R. System dynamics, a unified approach. Wiley, New York, 2nd edition, 1990.

10. Khalil, H. K. Nonlinear Systems. MacMillan, New Jersey, 2 edition, 1992.

11. Nijmeijer, H., van der Schaft, A. J. Nonlinear Dynamical Control Systems. Springer-Verlag, 1990.

12. Ortega, R., Loría, A., Nicklasson, P. J., Sira-Ramírez, H. Passivity-Based Control of Euler-Lagrange Systems. Springer-Verlag, 1998.

13. van der Schaft, A. J. $L_{2}$-gain and Passivity Techniques in Nonlinear Control. Springer-Verlag, 2000.

14. Yeh, T. Backstepping control in the physical domain. In Proceedings of the American Control Conference, 1999. 